\title{
We Asked the Experts: Direct Peritoneal Resuscitation: A Modern Adaptation of a Historical Technique
}

\author{
Woon Cho Kim ${ }^{1} \cdot$ Ronald B. Tesoriero ${ }^{1} \cdot$ Deborah M. Stein $^{1}$
}

Published online: 14 May 2020

(C) Société Internationale de Chirurgie 2020

Direct peritoneal resuscitation (DPR) has been increasingly recognized as a novel resuscitative adjunct to damage control surgery in patients with hemorrhagic or septic shock. Through the infusion of hypertonic fluid into the peritoneum, DPR has been demonstrated to promote visceral perfusion, blunt systemic inflammatory response, improve rates of abdominal closure, and improve clinical outcomes in trauma and acute general surgical patient populations [1]. We aim to offer a brief overview of DPR and practical tips for the general surgeons who wish to apply this technique into their practice.

\section{Historical description}

Peritoneal irrigation in a catastrophic abdomen is not an entirely new concept. Previously termed "continuous peritoneal lavage" or "postoperative peritoneal lavage," applying peritoneal irrigation into a septic abdomen beyond the index operation and during the resuscitative period has been well described, including Dr. John Ranson's approach to severe acute necrotizing pancreatitis [2]. The indications and patient population most likely to benefit from DPR remain ill defined. Additionally, the lack of a standardized protocol for type of solution, duration, and volume infused makes comparison across studies challenging [3].

Woon Cho Kim

wooncho.kim@ucsf.edu

1 Department of Surgery, University of California San Francisco, San Francisco, CA, USA

\section{Modern adaptation}

Multiple studies have demonstrated the benefit of DPR in hemorrhagic and septic shock. There has been a number of publications from University of Louisville on this subject, including a landmark clinical study by Smith and his colleagues [1]. Animal models in hemorrhagic shock suggest that DPR improves and sustains visceral organ blood flow even beyond the immediate resuscitation period compared to conventional resuscitative methods $[4,5]$. In addition to its direct peritoneal effects, DPR downregulates systemic inflammatory cytokine levels and pro-inflammatory mediators, resulting in improved systemic perfusion [5].

DPR in patients undergoing damage control surgery from hemorrhagic shock leads to higher rate of primary fascial closure and shortened time to definitive fascial closure [6]. When applied to patients undergoing damage control surgery from abdominal sepsis, DPR not only achieved faster fascial closure, but also shortened the length of ICU stay and ventilator-dependent days and decreased intraabdominal complications [1].

\section{Practical tips}

DPR can be applied to patients undergoing damage control surgery for a wide range of acute surgical emergencies. Smith and his colleagues describe a standardized protocol for DPR in patients undergoing damage control surgery with concurrent temporary abdominal closure [1]. First, a $19 \mathrm{~F}$ round Blake drain tubing is inserted through the abdominal wall and internally placed near the base of the mesentery. A vacuum pack abdominal closure device is applied using a fenestrated sterile bag, surgical towel, and occlusive dressing, with another drain for drainage (Blake 
Fig. 1 Step-by-step illustration of applying a direct peritoneal resuscitation (DPR) dressing following a damage control surgery in a patient who underwent small bowel resection for bowel ischemia. a Two 19F Blake drains are placed bilaterally in the paracolic gutters; b a large sterile towel drape with perforations is inserted into the peritoneal cavity to allow drainage while protecting the viscera; c a surgical towel is layered over the drape; $\mathbf{d}$ one or two $28 \mathrm{~F}$ chest tubes are placed over the towel; e a generous size occlusive dressing (3 M Ioban is pictured here) is applied to hold suction

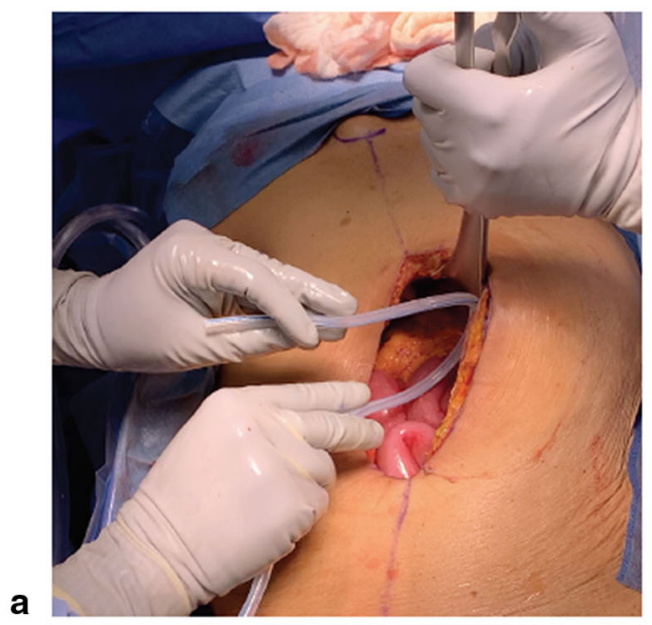

b
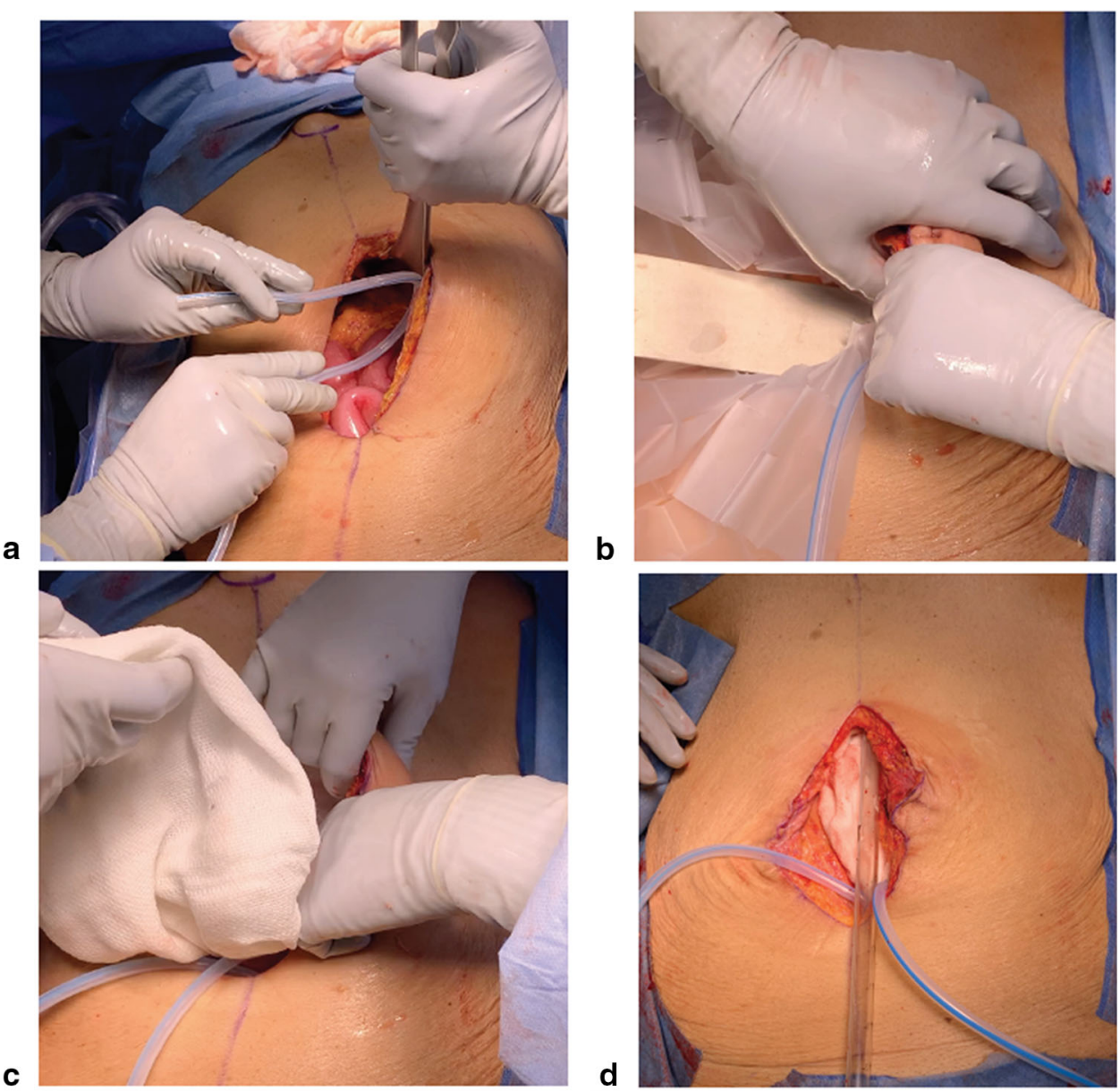

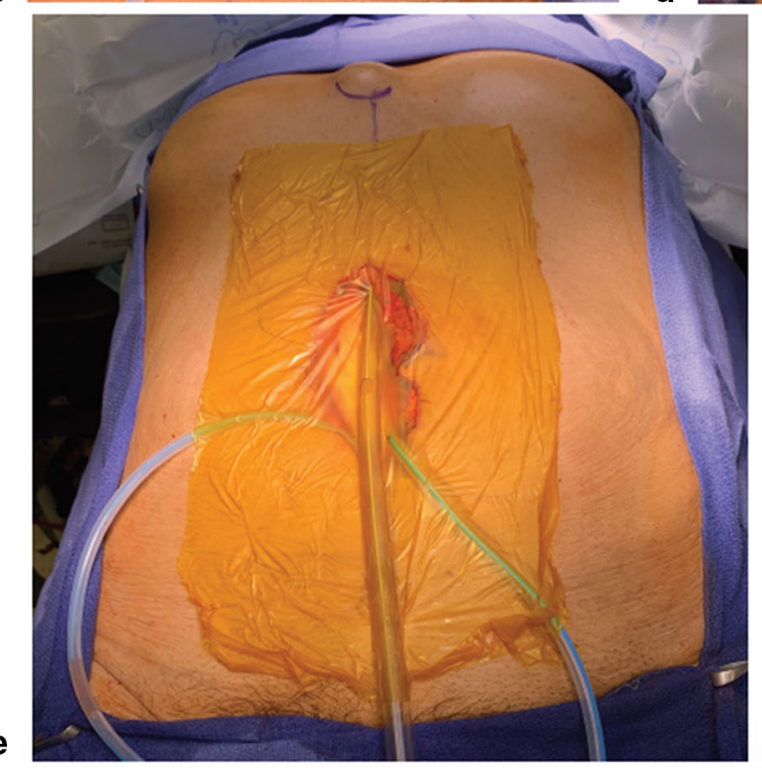

drain or chest tube). The chest tube is connected to continuous wall suction for drainage. Alternatively, a commercially available negative pressure wound management system can be used as the egress modality.
Once a good seal is achieved, commercially available $2.5 \%$ glucose-based peritoneal dialysis solution is infused at $800 \mathrm{cc} / \mathrm{h}$ for the first hour, followed by a continuous lavage at $400 \mathrm{cc} / \mathrm{h}$ [1]. The solution may be warmed to prevent worsening hypothermia. In our experience, it is 
advantageous to place two $19 \mathrm{~F}$ Blake drains for infusion and set the suction to $50-75 \mathrm{mmHg}$ to allow better distribution of the peritoneal fluid (Fig. 1). The authors' experience with DPR is preferentially in patients with an open abdomen; however, application of DPR in a closed abdomen has been described by insufflating the abdomen with smaller volume dialysate [4]. In either case, one must ensure that the equivalent volume of fluid instilled into the abdomen is evacuated to avoid acute compartment syndrome.

\section{How long should one continue DPR?}

DPR may be continued as long as necessary until definitive abdominal closure is achieved. DPR significantly shortens time to definitive abdominal closure compared to the conventional resuscitative group (5.9 days vs. 7.7 days), with reduced number of trips back to the operating room during their hospitalization [1].

\section{Can DPR be applied with abdominal packing for hemorrhage?}

DPR should be started after active hemorrhage has resolved, and the abdominal packing is removed. Delayed DPR, applied after conventional intravenous resuscitation, can be beneficial as it has been shown to reverse visceral hypoperfusion and vasoconstriction in hemorrhagic shock [7].

What type of solution should be used? Is there a role for antibiotic irrigation?

Most literature available on DPR to date is from the University of Louisville experience, which preferentially uses the $2.5 \%$ Delflex peritoneal dialysis solution
(Fresenius Medical Care North America, Waltham, MA, USA) [1, 5-7]. Other commercially available $2.5 \%$ glucose-based peritoneal dialysis solution with equivalent tonicity can be a safe substitute. Although isotonic fluids may be used, the efficacy of peritoneal dialysis solution in DPR stems from its hypertonic quality that reduces endothelial cell swelling and promotes capillary blood flow of visceral organs [7]. Adding antibiotic to the DPR solution has not been studied, but is unlikely to add benefit in an abdomen with adequate source control.

\section{References}

1. Smith JW, Garrison RN, Matheson PJ et al (2014) Adjunctive treatment of abdominal catastrophes and sepsis with direct peritoneal resuscitation: indications for use in acute care surgery. J Trauma Acute Care Surg 77(3):393-398

2. Ranson JH, Spencer FC (1978) The role of peritoneal lavage in severe acute pancreatitis. Ann Surg 187(5):565-575

3. Dong Z, Petrov MS, Xu J et al (2010) Peritoneal lavage for severe acute pancreatitis: a systematic review of randomised trials. World J Surg 34(9):2103-2108. https://doi.org/10.1007/s00268-0100665-3

4. Weaver JL, Smith JW (2016) Direct peritoneal resuscitation: a review. Int J Surg 33:237-241

5. Zakaria ER, Hurt RT, Matheson PJ et al (2003) A novel method of peritoneal resuscitation improves organ perfusion after hemorrhagic shock. Am J Surg 186(5):443-448

6. Smith JW, Garrison RN, Matheson PJ et al (2010) Direct peritoneal resuscitation accelerates primary abdominal wall closure after damage control surgery. J Am Coll Surg 210(5):658-664

7. Zakaria ER, Garrison RN, Kawabe T et al (2005) Direct peritoneal resuscitation from hemorrhagic shock: effect of time delay in therapy initiation. J Trauma 58(3):499-506

Publisher's Note Springer Nature remains neutral with regard to jurisdictional claims in published maps and institutional affiliations. 